\title{
IMPACTO AMBIENTAL PROVOCADO POR EL INADECUADO USO DE FERTILIZANTES QUIMICOS EN CULTIVOS DE MAÍZ
}

AUTORES: $\quad$ Franklin Ecuador Jaime Calderón $1^{1}$

Jorge William Castro Guerra $2^{2}$

Diomedes Augusto Orlando Lucio $3^{3} \ldots$

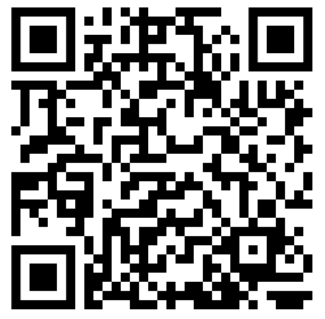

\section{DIRECCIÓN PARA CORRESPONDENCIA: (frankjcalderon46@yahoo.com )}

Fecha de recepción:

Fecha de aceptación:

\section{RESUMEN}

La presente investigación, tuvo como objetivos identificar la aplicación de contaminantes químicos, que usan los agricultores y la frecuencia de aplicación en el cultivo del maíz. Se utilizó la metodología de campo, se tomó muestras en fuentes de agua. Los resultados indican que se tiene entre 1500 y 2000 hectáreas sembradas de maíz y el promedio de producción en quintales por hectáreas esta entre 51 y 100, la calidad del suelo es regular. En la identificación de los contaminantes químicos de aguas superficiales, los resultados indican que el limite máximo permisible, de acuerdo a la norma del texto unificado de la legislación secundaria del ministerio del ambiente tomo a; para aguas de consumo humano y uso doméstico que únicamente requieren tratamiento convencional, aquí se puede ver que el resultado obtenido es menor 0.01000 , el paraquat presenta valores menor 0.1 y el glifosato así mismo presenta valores menor 0.001 , lo que indica que las aguas del río todavía no presentan una contaminación alta.

PALABRAS CLAVE: Ambiental; cultivo; contaminantes; fertilizantes.

\footnotetext{
${ }^{1}$ Ficha curricular del autor1: Ingeniero en Administración de Empresas Agropecuarias. Magister en Administración Ambiental, categoría G, docente investigador en la Unidad Educativa Fiscal "Enrique Guevara Galarza" Jipijapa Manabí Ecuador. E-mail: frankjcalderon46@yahoo.com.

${ }^{2}$ Ficha curricular del autor2 Ingeniero en Informática. Magister en Educación Informática Categoría G, docente investigador en la Unidad Educativa Fiscal "Coronela Filomena Chávez" Jipijapa Manabí Ecuador. E-mail: cjorgewilliam@yahoo.com.

${ }^{3}$ Ficha curricular del autor3 Ingeniero Agrónomo, docente Universidad Estatal del Sur de Manabí. Jipijapa Manabí Ecuador. E-mail: augusorlandi110@ hotmail.com.
} 
Autor1: Franklin E. Jaime Calderon, Autor2: Jorge W. Castro Guerra, Autor3: Diomedes A. Orlando Lucio

\title{
ENVIRONMENTALIMPACT FOR THE INADEQUATE USE OF CHEMICAL FERTILIZERS IN CULTIVATIONS OF THE CORN
}

\begin{abstract}
The objective of the present investigation was to identify the application of chemical contaminants, which farmers use and the frequency of application in the cultivation of corn. The field methodology was used, samples were taken from water sources. The results indicate that there are between 1,500 and 2,000 hectares planted with corn and the average production in quintals per hectare is between 51 and 100, the quality of the soil is regular. In the identification of surface water chemical contaminants, the results indicate that the maximum permissible limit, according to the standard of the unified text of the secondary legislation of the Ministry of the Environment, took into account; for waters for human consumption and domestic use that only require conventional treatment, here it can be seen that the result obtained is less than 0.01000 , the paraquat has values less than 0.1 and the glyphosate itself has values less than 0.001, which indicates that the waters of the river still do not present a high contamination.
\end{abstract}

KEYWORDS: Environmental - cultivation - contaminants - fertilizers. 


\section{INTRODUCCIÓN}

A nivel mundial el modelo de producción agrícola intensiva tiene la finalidad de aumentar los rendimientos de cultivos, utiliza insumos agrícolas como los fertilizantes químicos, plaguicidas y herbicidas, a pesar que los agroquímicos tienen efectos nocivos tanto para la salud de las personas como para el ambiente, los fertilizantes químicos y los insumos agrícolas aumentan la productividad en los primeros años que se aplican.

En la actividad agrícola e industrial asociada a un producto determinado (arroz, caña de azúcar), se utilizan una serie de recursos (energía, materias primas), y se producen una serie de desechos, los cuales producen un efecto sobre el medioambiente y que en ocasiones se desconoce. En el estudio de los impactos ambientales a partir de las herramientas de gestión ambiental, se tienen una serie de indicaciones normativas. (Llanes, 2006)

Por lo tanto, la situación actual de la problemática medio ambiental se caracteriza por la urgencia que ha adquirido su análisis y atención en la contemporaneidad, se manifiesta un amplio espectro de rasgos que gravitan en forma negativa en todos los confines de la sociedad y la economía planetaria, ha surgido un nuevo debate ético: el motivado por los cambios ambientales que, a escala global y local, afectan a toda la humanidad y amenazan su propia existencia.

El agotamiento de recursos y la liberación de contaminantes al ambiente han llegado a un extremo preocupante, que compromete la calidad de vida y la supervivencia de las generaciones presentes y futuras, así como el propio crecimiento económico, por la forma inadecuada en el manejo de los fertilizantes.

La protección del medio ambiente y la concepción del desarrollo sostenible implican un tipo de desarrollo en los campos productivos y sociales que satisfaga las necesidades básicas de la actual generación humana, sin poner en peligro las posibilidades de las sociedades venideras. Esto requiere de voluntades, decisiones y puesta en práctica 
Autor1: Franklin E. Jaime Calderon, Autor2: Jorge W. Castro Guerra, Autor3: Diomedes A. Orlando Lucio

de acciones políticas, económicas, científicas y educativas que se deben ver reflejadas en la responsabilidad del individuo, de la sociedad y de los estados frente a los problemas ambientales, para de esta forma contribuir a la Educación Ambiental en el presente siglo. (Alonso G \& Fontes, 2012).

En este sentido se puede apreciar que en Ecuador se potencia el debate sobre los vínculos entre actividad agrícola, comercio y ambiente; la internalización del concepto de desarrollo sostenible en la actividad agrícola productiva y comercial; así como la difusión de alternativas para conjugar los objetivos ambientales con los comerciales. En consecuencia, el objetivo a cumplir en esta investigación es contribuir a fortalecer los vínculos con organizaciones no gubernamentales, nacionales, organismos internacionales y los sectores privados para promover iniciativas que permitan comprender amplia y claramente los efectos que tienen las relaciones entre la actividad agrícola, el comercio y el ambiente en las expectativas del crecimiento económico, bienestar social y protección de los recursos naturales.

En el cantón Jipijapa, y en especial en la parroquia El Anegado, la actividad agrícola a la vez que es un motor impulsor de su desarrollo económico, fue en los últimos años su principal elemento contaminador, los recursos naturales utilizados para el cultivo del maíz, están afectados por el uso indiscriminado de agroquímicos para su producción, la deficiente concienciación y la continua utilización de estos productos en cada época de siembra, y no se evita el impacto negativo sobre el medio ambiente.

\section{DESARROLLO}




\section{MATERIALES Y MÉTODOS}

La presente investigación se desarrolló en la parroquia El Anegado del cantón Jipijapa durante seis meses desde enero, hasta agosto de 2014 . La temperatura promedio osciló entre: $21^{\circ} \mathrm{C}$ a 24,5 ${ }^{\circ} \mathrm{C}$, la altura: 800 metros sobre el nivel del mar; factores que incidieron en los resultados a del análisis físico - químico.

\section{RECURSOS EMPLEADOS EN LA INVESTIGACIÓN}

Recursos Humanos: investigadores, encuestadores, ingeniero químico.

Recursos físicos: encuestas y mapas de reconocimiento.

El universo estuvo conformado por 132 productores de maíz de la parroquia El Anegado, registrados en el Ministerio de Agricultura, Ganadería, Acuacultura y Pesca (MAGAP).

Se aplicó la siguiente fórmula para obtener la muestra de poblaciones finitas:

$$
n=\frac{N^{*} Z_{a}^{2} p^{*} q}{d^{2} *(N-1)+Z_{a}^{2}{ }^{*} p^{*} q}
$$

Donde:

$\mathrm{N}=$ Total de la población

132

$Z \alpha=1.96$ al cuadrado (si la seguridad es del $95 \%$ )

1,96

$\mathrm{p}=$ proporción esperada (en este caso $5 \%=0.05$ )

0,05

$\mathrm{q}=1-\mathrm{p}$ (en este caso $1-0.05=0.95)$

0,95

$\mathrm{d}=$ precisión (en su investigación use un $6 \%$ ).

0,06

$\mathbf{n}=$

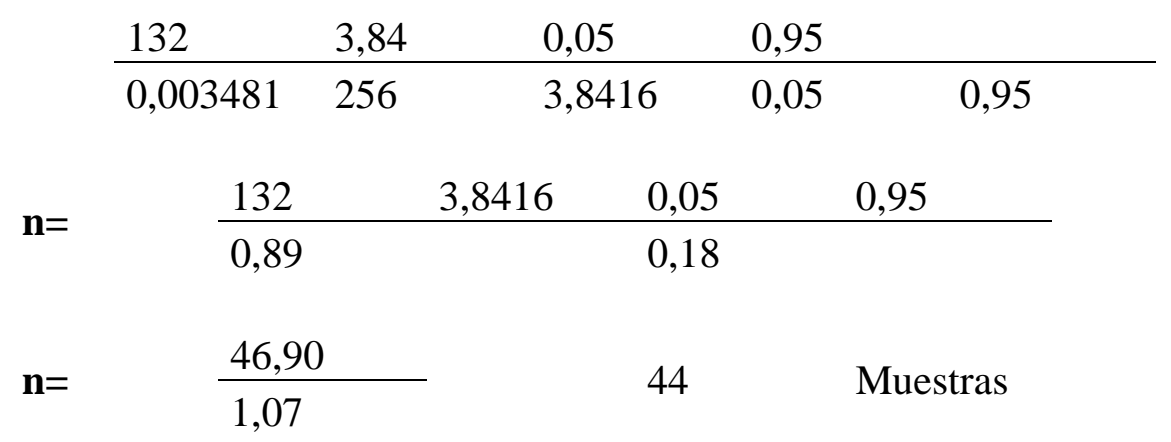


Autor1: Franklin E. Jaime Calderon , Autor2: Jorge W. Castro Guerra, Autor3: Diomedes A. Orlando Lucio

A partir del cálculo de la muestra, se trabajó con 44 productores que representan el 33,3\% de los diferentes recintos de la parroquia El Anegado, lo cual resulta una muestra confiable.

En esta investigación predomina el método bibliográfico documental y de campo.

\section{RESULTADOS}

El estudio del impacto ambiental por el inadecuado uso de fertilizantes químicos en el cultivo del maíz en la parroquia El Anegado, se desplegó a partir del cumplimiento de los siguientes objetivos:

\section{Objetivo 1. Determinar los fertilizantes que usan los agricultores, $y$ frecuencia de aplicación}

Para conocer en toda su magnitud el problema de impacto ambiental, debido a los fertilizantes químicos, en la parroquia El Anegado, se identificó los tipos (tabla 1) la frecuencia de aplicación (tabla 2), y su grado de peligrosidad en el cultivo de maíz (tabla 3). Mediante encuestas estructuradas a los productores.

\section{¿Qué tipos de fertilizantes usted utiliza en la producción de maíz?}

\section{Tabla $\mathbf{N}^{\circ} 1$. Tipos de fertilizantes que se utiliza en la producción de maíz}

\begin{tabular}{|l|r|r|}
\hline \multicolumn{1}{|c|}{ Alternativas } & Encuestas & Porcentaje (\%) \\
\hline QUÍMICO & 41 & 93 \\
\hline ORGÁNICO & 2 & 5 \\
\hline SIN RESPUESTA & 1 & 2 \\
\hline TOTAL & $\mathbf{4 4}$ & $\mathbf{1 0 0}$ \\
\hline
\end{tabular}

Fuente: Agricultores maiceros de El Anegado

Elaborado por: Autores de la investigación

Se refleja que la mayoría de los productores utilizan agroquímicos, lo que podría ocasionar a la larga una contaminación del suelo y de agua causando un impacto negativo para estos recursos naturales. 
¿Con qué frecuencia utiliza fertilizantes químicos en sus cultivos del maíz?

Tabla $\mathbf{N}^{\circ}$ 2. Frecuencia con que utiliza los fertilizantes químicos

\begin{tabular}{|l|r|r|}
\hline \multicolumn{1}{|c|}{ Alternativas } & Encuestas & Porcentaje (\%) \\
\hline SIEMPRE & 40 & 91 \\
\hline A VECES & 3 & 7 \\
\hline NUNCA & 1 & 2 \\
\hline TOTAL & $\mathbf{4 4}$ & $\mathbf{1 0 0}$ \\
\hline
\end{tabular}

Fuente: Agricultores maiceros de El Anegado

Elaborado por: Autores de la investigación

Es evidente que la alternativa "siempre" predomina en este proceso, lo que puede evidenciar un uso indiscriminado de estos productos y una baja concienciación del efecto que produce.

¿Tiene conocimiento del grado de peligrosidad del uso de los agroquímicos?

Tabla $\mathrm{N}^{\circ}$ 3. Conocimiento del grado de peligrosidad del uso de los agroquímicos

\begin{tabular}{|l|r|r|}
\hline \multicolumn{1}{|c|}{ Alternativas } & Encuestas & Porcentaje (\%) \\
\hline SI & 42 & 95 \\
\hline NO & 2 & 5 \\
\hline TOTAL & $\mathbf{4 4}$ & $\mathbf{1 0 0}$ \\
\hline
\end{tabular}

Fuente: Agricultores maiceros de El Anegado

Elaborado por: Autores de la investigación

Se concluye que los agricultores conocen el peligro del uso de los fertilizantes químicos pero lo usan para incrementar los rendimientos por hectárea.

¿Conoce usted las bondades del uso de fertilizantes orgánicos en la producción de maíz?

Ante esta pregunta el $82 \%$ expresaron que si lo conocían, lo que evidencia que existe un grado de conocimiento de este tema aceptable. 
Autor1: Franklin E. Jaime Calderon , Autor2: Jorge W. Castro Guerra, Autor3: Diomedes A. Orlando Lucio

\section{¿Cuál es la razón fundamental por la que usted utiliza fertilizantes químicos, para la producción de maíz?}

Las razones que argumentaron están relacionadas con:

La mayor productividad (80\%)

El desconocimiento de otros métodos $(13 \%)$

El poco incentivo para la producción de productos agroecológicos. $(6 \%)$.

Otras $(1 \%)$

\section{Objetivo 2. Identificar los contaminantes químicos de aguas superficiales.}

Se realizó el análisis físico-químico del agua en el rio Juan Montalvo de la parroquia El Anegado, en el laboratorio especializado Grupo Químico Marcos Cía. Ltda. (Ver anexo \# 1), el tipo de muestreo fue integrado y se utilizó la norma del Texto Unificado de la Legislación Secundaria del Ministerio del Ambiente TULSMA y los límites máximos permisibles para aguas de consumo humano y uso doméstico que únicamente requieren tratamiento convencional.

Los análisis indicaron que aun cuando existe presencia de compuestos químicos dañinos debido al uso de fertilizantes químicos, están en un porcentaje que no excede los límites máximos permisibles (LMP), de acuerdo a la norma (TULSMA), № 3.516, R.O. Edición Especial No.2 del registro oficial, con fecha 31 de marzo del 2003. Texto reglamentario bastante amplio de la normativa ecuatoriana vigente en la Ley de gestión ambiental, que nos indica que aún estamos a tiempo de evitar un problema ambientalista. 


\section{CONCLUSIONES}

Los productores de maíz de la parroquia El Anegado del cantón jipijapa de Manabí, República del Ecuador utilizan fertilizantes químicos, aun cuando tienen conocimiento de las bondades de fertilizantes orgánicos en los cultivos, a pesar de conocer la peligrosidad que significa el uso de estos, los agricultores encuestados obtienen en su mayoría el agua a través de las tuberías es decir reciben agua entubada y la calidad esta entre buena y regular; las enfermedades más comunes es de tipo respiratoria y de la piel. La calidad de los suelos donde siembran maíz es de tipo regular, además tienen suelos erosionados y el deterioro de los suelos donde se practica la agricultura se debe al uso de fertilizantes químicos.

En la identificación de los contaminantes químicos de aguas superficiales, los resultados del laboratorio indican que el (LMP) de acuerdo a la norma: TULSMA, aquí se puede ver que el resultado obtenido es menor a 0.01000 , el Paraquat presenta valores menor a $0.1 \mathrm{y}$ el glifosato así mismo presenta valores menor a 0.001, lo que indica que las aguas del río del Recinto Juan Montalvo de la parroquia El Anegado todavía no presentan una contaminación alta.

Podemos concluir que es necesario hacer un plan de manejo ambiental (PMA), que contribuya a la concienciación del uso de productos orgánicos en el cultivo de maíz y buscar mercados en los que se cotice mejor los productos agroecológicos, para incentivar la producción de los mismos y de esta forma disminuir el uso de fertilizantes químicos. 


\section{ANEXO 1}

\section{RESULTADOS DE LOS ANÁLISIS REALIZADOS}

\begin{tabular}{|c|c|c|c|c|c|c|}
\hline 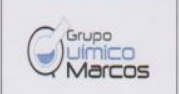 & & \multicolumn{3}{|c|}{$\begin{array}{l}\text { INFORME DE ENSAYOS } \\
\text { No. 41702-1 }\end{array}$} & & \\
\hline \multicolumn{5}{|c|}{ 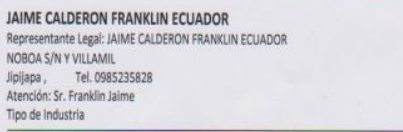 } & \multicolumn{2}{|c|}{ Uayaquil, 18 DE NOVEMMBRE DEL 2014} \\
\hline $\begin{array}{l}\text { Fecha, Hora y lugar de Muestreo: } \\
\text { Fecha y Hora de Recepción: } \\
\text { Punto e ldentificacion de la Muestra: } \\
\text { Norma Ténica de muestreo: } \\
\text { Matriz de la muestra: } \\
\text { Muestreado por: } \\
\text { Muestreador: } \\
\text { Tipo de Muestreo: } \\
\text { Temperatura de muestreo: }\end{array}$ & \multicolumn{6}{|c|}{ 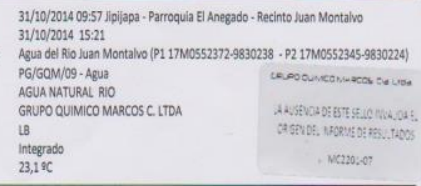 } \\
\hline \multicolumn{7}{|c|}{ 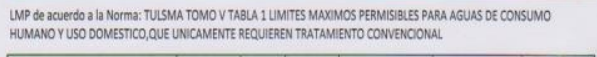 } \\
\hline Pardmetro & Resultado & $U K=2$ & Unidades & IMP & Metoodo Analitico & - Analizado \\
\hline $\begin{array}{l}\text { CONPONENTIS ORGaNicos: } \\
\text { Pesticidas } \\
\text { Organofosforados:Atrazina (1) }\end{array}$ & $<0,01000$ & - & $\mathrm{m}_{8} /$ & - & 6640 B & 24/11/2014 $\mathrm{Mm}$ \\
\hline Paraquat (1) & $<0,1$ & - & บell & - & $6630 \mathrm{C}$ & oa/11/20140 or \\
\hline Pardmetro & Resultaso & $u k=2$ & Unidades & LMP & Métooso Analisice & Analizado \\
\hline $\begin{array}{l}\text { Asicoquivicos: } \\
\text { Gifiosato (1) }\end{array}$ & $<0,001$ & - & man & 2000 & 66518 & Oa/11/2004 or \\
\hline
\end{tabular}

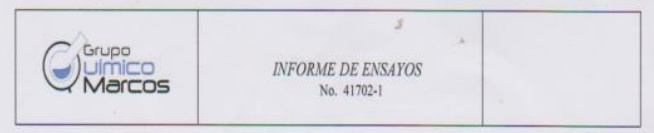

JAIME CALDERON FRANKUN ECUADOR
Representante Legal: IAME CALDRON FRANKUN ECUADOR

NOBOA S/N Y VLLAMIL

Sipjipa, Tel. 0985235828

Tipo de industria

Fecha, Hora ylugar de Muestreo: $\quad 31 / 10 / 2014$ 09:57 I.jpjapa - Parroquia El Anegado - Recinto Juan Montalivo

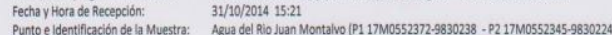

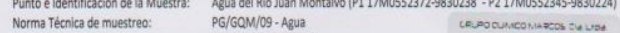

Norma Tecrica de muestreo.

Muestreado por.

Muestreador:

Tipo de Muestreo:

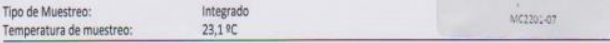

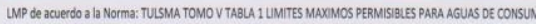

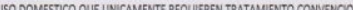
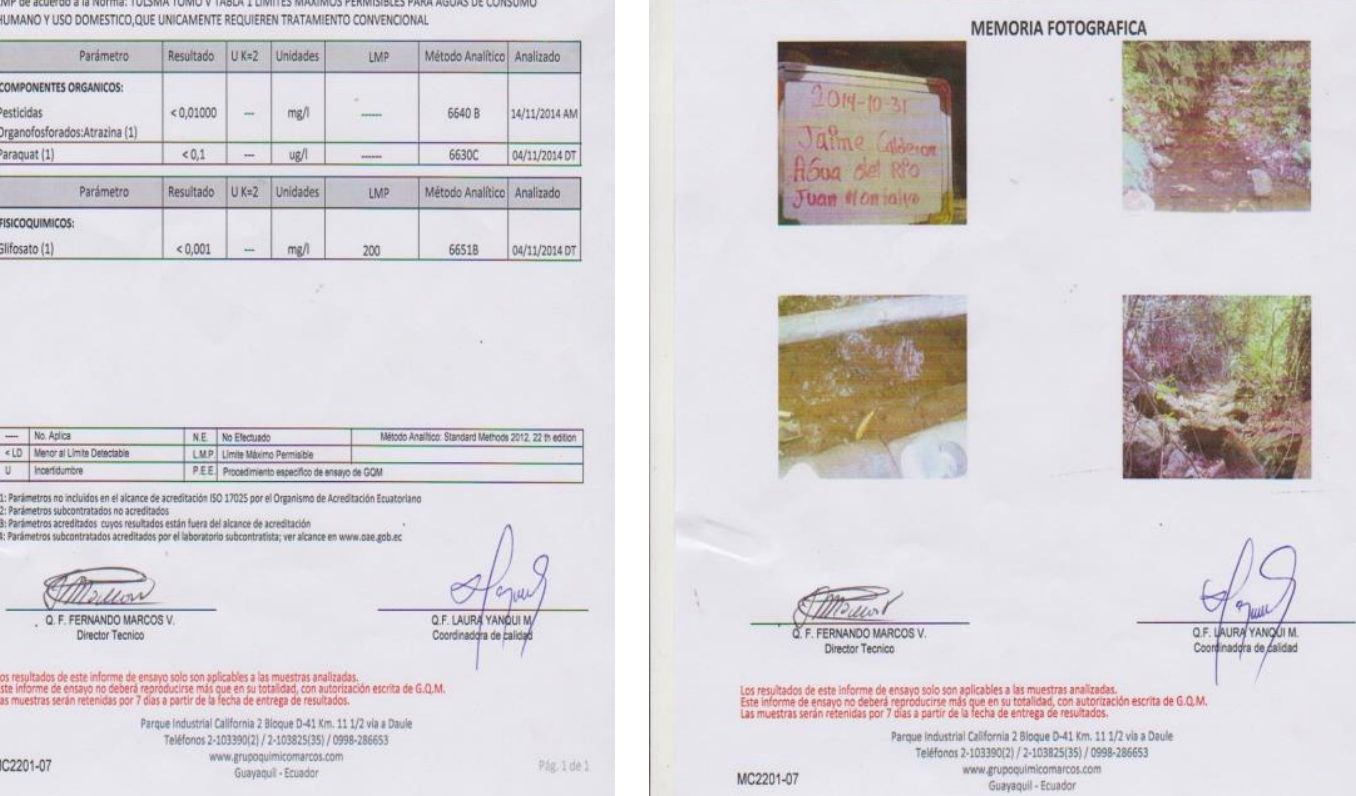

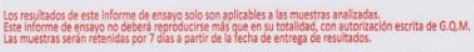

MC2201-07

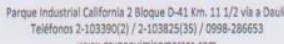

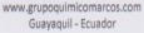




\section{REFERENCIAS BIBLIOGRÁFICAS}

ATDSR. (2007). La toxicidad del plomo. Esta página fue revisada el 20 de agosto del 2007. Agencia para Sustancias Tóxicas y el Registro de Enfermedades ATDSR, 4770 BufordHwy NE, Atlanta, GA 30341. Disponible en: http://www.atsdr.cdc.gov/es/csem/plomo/es_pb$\underline{\text { normas.html }}$

BRAÑES, Raúl (2000). El acceso a la justicia ambiental en América Latina. Derecho Ambiental y Desarrollo Sostenible, Pág.45. Serie de Documentos de Derecho Ambiental núm. 9. PNUMA. PROFEPA. México.

CarguaJ. ; Mite, F. ; Carrillo, M. y Durango, W. (2010). Determinación de las formas de Cu, $\mathrm{Cd}, \mathrm{Ni}, \mathrm{PbY} \mathrm{Zn}$ y su Biodisponibilidad en suelos agrícolas del litoral ecuatoriano. XII Congreso Ecuatoriano de la Ciencia del Suelo. Carrera de Ingeniería Agropecuaria. Universidad Tecnológica Equinoccial-Santo Domingo. Departamento Nacional de Manejo de Suelos y Aguas. Estación Experimental Tropical Pichilingue (INIAP). Santo 17-19 Domingo, 17-19 de noviembre del 2010. PDF. p.1- 17. Disponible en: http://www.secsuelo.org/XIICongreso/Simposios/Relase/Ponencias/1.\%20Cargua\%20\%20Determinacion\%20cadmio.pdf

Carrera, J. (1994). Evaluación del contenido de Cadmio en el sistema suelo-cacao de varias zonas del Ecuador. Tesis Ing. Agrónomo. Universidad Agraria del Ecuador. Facultad de Ciencias Agrarias. Guayaquil, EC. p: 53-65.

Carrillo, M. (2003). Formas y características de materiales pesados y su disponibilidad, absorción dinámica y movilidad en los suelos de Ecuador. Tesis Mag Sc. Universidad Federal de Vicosa. Departamento de Solos. Vicosa, BR. p: 56. 
Autor1: Franklin E. Jaime Calderon , Autor2: Jorge W. Castro Guerra, Autor3: Diomedes A. Orlando Lucio

D. S. MACKINNON AND B. FREEDMAN, (1993), "Effects of Silvicultural Use of the Herbicide Glyphosate on Breeding Birds of Regenerating Clearcuts in Nova Scotia, Canada", Journal of Applied Ecology, Vol. 30, No. 3 pp. 395-406.

INIAP (Instituto Nacional Autónomo de Investigaciones Agropecuarias, EC); PROMSA (Programa de Modernización Agropecuaria, EC). (2003). Determinación de metales contaminantes en cultivos de exportación y su repercusión sobre la calidad de los mismos. Quevedo, EC. p: 60. (en línea). Consultado 14 jun. 2009. Disponible en: http://www.mag.gov.ec/promsa /Resumen\%20IG-CV-023.htm.

INIAP (Instituto Nacional Autónomo de Investigaciones Agropecuarias, EC), (2009). Informe Técnico del Proyecto "Monitoreo de presencia de cadmio en almendras de cacao, suelos y aguas y alternativas de control". Estación Experimental Tropical Pichilingue-Quevedo, EC. p: 16.

POJMAN, L. P. (2005). Environmental Ethics. Thompson - Wadsworth Eds., Toronto, Ontario, p: 678 .

Saavedra. C. (2009). "El manejo, protección y conservación de las fuentes de agua y recursos naturales" - Cartilla educativa. Una publicación del PROGRAMA DE GOBERNABILIDAD PARA EL DESARROLLO TERRITORIAL SOSTENIBLE CONCERTAR, realizada en el marco del "III Concurso Nacional del Agua". Ministerio del Agua, a través del Vice ministerio de Recursos Hídricos y Riego. PROAGRO - GTZ. CONCERTAR - INTERCOOPERATION - COSUDE. La Paz, Bolivia. Primera edición. ® Octubre, 2009. Pdf. p: 22-33.

VILLALOBOS Francisco, MATEOS Luciano (2009). Fitotecnia: bases y tecnologías de la producción agrícola. Mundi-Prensa Libros, p: 496. 